\title{
Estratégia\&Negócios
}

ISSN 1984-3372

http://www.portaldeperiodicos.unisul.br/index.php/EeN/

\section{FAIR TRADE (COMÉRCIO JUSTO) COMO UM 'TÓPICO QUENTE” INTERNACIONAL E SUA ABORDAGEM NO BRASIL}

FAIR TRADE: AS AN INTERNATIONAL HOT TOPIC AND ITS APPROACH IN BRAZIL

\section{José Carlos Lazaro Silva-Filho}

Doutorado em Planejamento Ambiental pela Technische Universität-Berlin - Alemanha. Mestrado em Administração na área de Gestão de C\&T pelo PPGA da Universidade Federal do Rio Grande do Sul.

Professor Adjunto da Universidade Federal do Ceará.

E-mail: silvafilhode@yahoo.com.br

Flavio Leandro Batista de Moura Cantalice

Universidade Federal do Ceará.

E-mail: lazaro@ufc.br

Recebido em 03/07/2011. Aprovado em 06/09/2011. Disponibilizado em 03/12/2011.

Avaliado pelo Sistema double blind review

R. eletr. estrat. neg., Florianópolis, v.4, n.2, p. 223-244, jun./dez. 2011

http://portaldeperiodicos.unisul.br/index.php/EeN/index
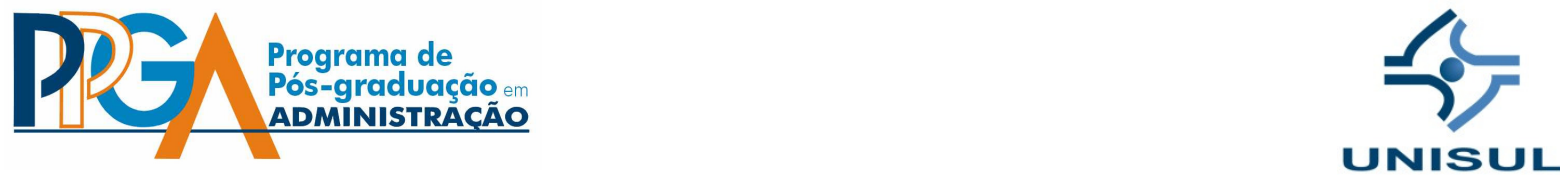

CCopyright 2008 UNISUL-PPGA/Estratégia e Negócios. Todos os direitos reservados. Permitida citação parcial, desde que identificada a fonte. Proibida a reprodução total. Em caso de dúvidas, consulte o editor:

ademar.unisul@gmail.com; (48) 3229-19 


\section{RESUMO}

Este artigo tem como objetivo analisar bibliometricamente o desenvolvimento do conceito de Fair Trade na literatura internacional e nacional, identificando a emergência do tema e analisando possíveis "barreiras" do tema no contexto nacional. Para isto foram feitas duas pesquisa bibliométricas, um na base "Web of Science" da ISI e outra nas publicações nacionais (anais e revistas). Embora usando uma análise bibliométrica tenha se classificado o tema com "emergente", isto é um "hot topic" (termo bibliométrico cunhado por Banks, 2006os autores perceberam uma defasagem considerável da abordagem do tema no Brasil. Após esta análise bibliométrica busca-se propor uma justificativa pela defasagem tanto na prática quanto nas discussões científicas pode ser melhor explicada quando utiliza-se uma abordagem teórica emergente da ciência social francesa, chamada de "qualidade e convenções", para entender a coordenação das redes de agronegócios. Nesta perspectiva pode-se afirmar que no Brasil o comércio justo ainda se encontra inscipiente como uma "convenção cívica" (moral), sendo que os casos que estão se desenvolvendo estão uma utilizando já uma "convenção de mercado", assimilando conceitos de redes alimentares de países desenvolvidos.

Palavras-Chave: fair Trade. Convetion Theory. Bibliometria.

\section{INTRODUÇÃO}

A globalização econômica nas últimas três décadas permitiu a diversidade de fornecimento de produtos para cadeias de suprimentos em qualquer parte do mundo aumentando a diversidade de produtos disponíveis ao consumidor no varejo, isto é no final das cadeias de suprimento. Paralelamente, neste mesmo período houve uma ascensão de preocupação da sociedade com questões ambientais e relacionadas a ética dos processos 
inerentes a obtenção do produto, seja da extração ou do trabalho envolvido na elaboração e disponibilização do produto.

A confluência do aumento da diversidade de produtos disponíveis de origens variadas com preocupação mais ativa de um grupo de consumidores em relação a seu próprio consumo cria um ambiente propício para uma crescente exigência de informação sobre as procedências e os processos dos produtos disponibilizados.

Neste ponto, produtores que vem praticando uma produção mais ambientalmente correta (quanto ao seu impacto total sobre a sociedade) ou socialmente correta (isto é dentro de padrões éticos financeiros aceitos globalmente, por exemplo cumprindo legislações locais trabalhistas e princípios gerais da Organização Mundial do Trabalho, OMT) podem encontrar uma demanda diferenciada, capaz inclusive de compreender que tais práticas elevam o custo de produção a um custo real (que inclui as externalidades ambientais e encargos sociais mínimos, muitas vezes ).

Dentre as práticas usuais de alinhamento de processos e estas demandas estão a de produtos orgânicos, de baixo impacto ambiental em seus processos, e aqueles desenvolvidos em arranjos produtivos mais socialmente justos, isto é com uma distribuição do lucro "mais justa" frente ao esforço e trabalho para sua obtenção. Estes últimos tem se agrupado em um conceito que vem sendo chamado de "Fair Trade", traduzido literalmente como "Comércio Justo".

Esta prática da exigência organizacional sobre os processos dos seus fornecedores está em ascensão no cenário internacional. E, Apesar de as vendas de Comércio Justo não estarem acima de $0,01 \%$ do volume total de comércio mundial (BECCHETTI; HUYBRECHTS; 2008), o índice de crescimento faz com que o Comércio Justo seja um importante fenômeno mundial. Segundo Raynolds (2002) o valor total negociado no mercado de Comércio Justo estava, até 2002, avaliado em 400 milhões de dolares crescendo cerca de $30 \%$ ao ano. Já nesta época as redes de Comércio Justo envolviam 800 organizações de produtores em 45 países em desenvolvimento (do "Sul"). Raynolds (2002) ainda sugere que, enquanto o sucesso de mercado do Comércio Justo é imprevisível, sua verdadeira importância não está na sua "parcela do mercado", que presumivelmente será sempre relativamente pequena, mas usa importância está na sua habilidade de criar vínculos entre o R. eletr. estrat. neg., Florianópolis, v.4, n.2, p. 223-244, jun./dez. 2011 
produtor e o consumidor e derrubar a divisão norte/sul. O índice de crescimento total do Comércio Justo no mundo, de acordo com o relatório anual da Fair Trade Label Organization (FLO, 2009), foi de $10 \%$ em 2008, com índices de crescimento individuais em alguns países impressionantes, como o crescimento de $75 \%$ da Suécia.

Este crescimento da prática internacional começa a ser notado também na produção científica sobre o tema, que leva a supor que este possa vir a ser considerado um "tópico quente" (hot topic), conforme classificação proposta por Banks (2006) em seus estudos bibliométricos.

Este artigo tem como objetivo identificar o estado da arte sobre o tema, desenvolvendo uma análise bibliométrica internacional e nacional; discutindo um possível descolamento das práticas de Comércio Justo e dos conceitos em desenvolvimento especificamente no caso brasileiro.

Para a análise bibliométrica internacional foi utilizado o catálogo eletrônico da THOMSON ISI (Web of Science) destacando-se algumas obras, e utilizando o método $\boldsymbol{h}-\boldsymbol{b}$ de Banks (2006) para a análise das citações recebidas. Para a análise bibliométrica nacional foram pesquisados trabalhos em eventos e revistas escolhidos por acessibilidade na área de administração (ou disponibilidade dos CDs de Anais ou periódicos com livre acesso), restringindo-se aos último 3 anos visto mesmo ter sido encontrado poucos artigos já nestes anos - e estes com nenhuma citação ou referência a textos nacionais anteriores

\section{CONCEITUANDO COMÉRCIO JUSTO OU “FAIR TRADE"}

Ao se criar a expressão internacional "Fair Trade" teve-se como idéia diferenciar e estimular, são iniciativas de comércio justo. Este teria como objetivo fornecer a garantia aos compradores de determinados produtos, que estes foram produzidos de maneira ética, levando em conta aspectos de como os produtores e os trabalhadores produzem ou plantam, incluindo alguns critérios sociais e ambientais. Apesar do conceito de Comércio 
Justo não ser consensual, algumas organizações ligadas ao seu desenvolvimento propõe definições que conceitualmente se aproximam

De acordo com a Fair Trade Labelling Organization International (FLO), comércio justo é "uma abordagem alternativa ao comércio convencional e é baseado em uma parceria entre produtores e consumidores, oferecendo aos produtores negócios melhores e permitindo que eles melhorem suas vidas e planejem seus futuros" (FLO, 2010).

$\mathrm{Na}$ busca de uma definição mais ampla a rede informal das quatro principais organizações de Comércio Justo (Fair Trade Labelling Association, International Fair Trade Association, Network European Workshops e European Fair Trade Association ) sob o anacronismo de FINE propõem o seguinte conceito:

Fair Trade é uma parceria comercial, baseada em diálogo, transparência e respeito, que procura maior equidade no comércio internacional. Ele contribui com o desenvolvimento sustentável oferecendo melhores condições de comércio, e ao garantir os direitos dos produtores e trabalhadores marginalizados, especialmente no sul. As organizações de Fair Trade (apoiadas pelos consumidores) estão engajadas ativamente em ajudar os produtores, aumentar a conscientização e fazer campanhas para mudança nas regras e na prática do comércio internacional (BECCHETTI; HUYBRECHTS, 2004; EFTA, 2010).

Já de acordo com Adams e Raisborough (2008), Comércio Justo seria apenas uma de várias formas de consumo éticas, tais como de cuidados com os animais e turismo sustentável, e é o exemplo primário de consumo ético. Consumo ético pode ser definido como qualquer prática de consumo na qual compromisso explícito em relação a outros distantes ou ausentes é uma importante dimensão no significado da atividade dos atores envolvidos (ADAMS; RAISBOROUGH , 2008)

A idéia que se tem com as definições dadas é que Comércio Justo é um conceito multidimensional. Enquanto muitos concordam com o caráter multidimensional do Comércio Justo, Beccheti e Huybrechts (2008) acreditam que seus elementos constituintes podem ser divididos em quatro categorias principais: 


\section{Comércio}

A primeira é o comércio (Trade), já que, ainda que justo (Fair), ainda estamos falando de comério. Os operadores de Comércio Justo conduzem diversas atividades de mercado, tais como importação, transformação e distribuição, similares a qualquer atividade comercial. A posição de comércio desses operadores, no entanto, pode variar, de um modo de produzir mais desenvolvimento no Sul (países em desenvolvimentos) a um objetivo próprio. Quando se analisa o produto do Comércio Justo, a dimensão "comércio" pode ser assimilada ao caráter material do produto. De fato, não é o próprio produto que é “justo", já que nada distingue fisicamente uma banana do Fair Trade de uma convencional, mas sim as características invisíveis ligadas às condições nas quais o produto foi produzido e comerciado.

\section{Justiça}

A dimensão de "justo" constitui o específico e poderoso (ainda que invisível) atributo do Comércio Justo, diferenciando-os dos demais produtos (NICHOLLS; OPAL, 2005, BECCHETI; HUYBRECHTS, 2008). Essa característica de "justo" é ligada tanto aos produtores quanto aos importadores dos produtos de Comércio Justo. Enquanto os primeiros um número de critérios organizacionais (produtores de pequena escala, tomadas de decisão democráticas, nenhuma discriminação...) os últimos recebem direito a condições específicas de comércio (preço justo, premium social, pré-financiamento, relacionamento a longo prazo...). Pesquisas em Comércio Justo já tentaram determinar se o preço justo e suas outras ferramentas levam efetivamente a um melhor desenvolvimento socioeconômico para os produtores do que no caso do comércio convencional (por exemplo, levando a menor desigualdade do que o comércio convencional por aumentar a remuneração dos produtores).

Contra-argumentando Maseland e De Vaal (2002), afirmam que esse seria o caso somente quando a demanda do produto é inelástica (como as commodities) e os consumidores têm preferências de justiça. Entretanto, Becchetti e Huybrechts (2006) 
argumentam que a abordagem dos críticos é muito estreita e não leva em conta as características não monetárias do Comércio Justo, tais como pré-financiamento das produções, promoção da conscientização do consumidor, entre outras.

\section{Educação e Informação}

Comércio Justo também pode ser visto como uma ferramenta na orientação e conscientização dos consumidores. Alguns operadores da cadeia do Comércio Justo conduzem campanhas de informação e educação dirigidas aos consumidores. A intenção dessas campanhas é promover o Comércio Justo e, mais globalmente, educar os cidadãos e governos sobre hábitos de consumo que são mais respeitosos para o meio ambiente e para as pessoas, especialmente para os "produtores no Sul". Mesmo se todos os operadores não conduzissem atividades educacionais, pode-se assumir que os consumidores do Comércio Justo financiam parte dessas atividades através da compra.

\section{Regulamentação}

Nas origens do Comércio Justo, existia uma intenção de definir uma nova estrutura de regulamentação para trocas comerciais. Esta estrutura é primeiramente aplicada dentro das parcerias de Comércio Justo dentro de um número de critérios específicos. Ainda mais, essa estrutura de regulamentação ainda intenta infuenciar os fluxo principal das praticas comerciais nas trocas internacionais. O design e controle das normas específicas dentro do setor do Comércio Justo tem sido feita pricipalmente por organizações de Comércio Justo que não conduzem o comércio de commodities, mas com foco não criação e controle das normas de Comércio Justo (isso é referido como o processo de selos).

\subsection{ABORDAGENS POSSÍVEIS PARA A INTERPRETAÇÃO DA FORMAÇÃO DE CADEIAS DE FAIR TRADE}


As dimensões propostas anteriormente acabam por permitir diversas abordagens sobre o tema, partindo-se de clareza que seu objeto é uma cadeia (ou rede) que liga o produtor ao consumidor (RAYNOLDS, 2002). Sendo assim base teórica inicial é a abordagem de rede de commodities que tem seu pilar em Gereffi (1994). Muitas discussões tanto na área acadêmica quanto na ativista a respeito de redes agroalimentares segue uma análise de produção, distribuição e consumo baseada em commodities. Entretanto, nos anos recentes, literaturas de diversas áreas adotaram análises guiadas por similares, porém bastante variadas, conceitualizações das cadeias de commodities, sistemas de commodities, filières e cadeia de valores. Do ponto de vista ativista, infraestrutura de commodities e seu vocabulário são comumente usados para descrever os supostos perigos do sistema agroalimentar principal. Dentro do movimento do Comércio Justo, o discurso foca em como cadeias historicamente exploradoras entre produtor e consumidor podem ser reestruturadas baseadas em idéias de justiça e equidade (REYNOLDS, 2002).

Enraizada na política econômica tradicional, a abordagem de commodities analisa os processos relacionados de produção, processamento, envio, marketing e consumo incorporados em dada commodity ou grupo de commodities relacionadas. Diferentes estruturas dão foco a diferentes facetas das redes. Embasado em uma combinação das tradições anteriores, Gereffi (1994) esboça três facetas chave da cadeia global de commodities: a articulação de produtos e serviços em uma seqüência de atividades que adicionam valores; a configuração organizacional e espacial, ou territorialidade, das empresas que formam redes de produção e marketing; e as relações poder, ou estrutura governamental, determinando como os recursos são alocados durante a cadeia de commodity (GEREFFI, 1994; REYNOLDS, 2002).

Apesar das informações importantes dadas pela abordagem tradicional de commodities da economia política, Reynolds (2002) se baseia nos elementos das críticas dessa abordagem e propõe uma abordagem de economia política de commodities revisada, uma que retenha o foco na teoria e na práxis, sugerindo que os insights chave fornecidos por estudos culturais, ator-rede e abordagens das "convenções" podem ser utilmente compatíveis com a análise tradicional. Em particular, uma abordagem de commodity pode ser fortalecida através de contribuições na análise de atores e ações na área do consumo, R. eletr. estrat. neg., Florianópolis, v.4, n.2, p. 223-244, jun./dez. 2011 
facetas simbólicas e discursivas de rede de commodities e convenções competitivas organizando as redes de commodities (REYNOLDS, 2002) sendo esta uma tendência de abordagem que vem se confirmando conforme Renard (2003).

A teoria das convenções, baseada na escola francesa das ciências sociais (WILKINSON, 1997) explica o desenvolvimento do Comércio Justo desde seu início como uma "convenção cívica", com comércio alternativo coordenado pela sociedade até que ela se torne uma "convenção de mercado".

Atualmente os produtos de Comércio Justo chegam ao mercado, de duas formas, uma pelas Organizações de Comércio Alternativo (ATO's - do inglês Alternative Trade Organizations), que identificam os produtos, importam e comercializam através de uma variedade de canais como, em feiras de igreja, internet e lojas próprias, entre outras (JONES; COMFORT; HILLIER, 2004). A outra forma é via "Iniciativas Nacionais (IN) de Selos" onde os produtos Comércio Justo são vendidos em organizações comerciais tradicionais, sendo identificados pelo selo. As INs utilizavam selos diferentes para garantir ao consumidor que os produtos vendidos atendiam a padrões socioeconômicos justos. Para unificar os critérios internacionais de Comércio Justo e de adotar um sistema comum de certificação, em 1997, as INs fundaram a FLO. A FLO é uma organização independente responsável, por certificar os produtos e estabelecer os critérios de Fair Trade, seguindo práticas internacionais de monitoramento externo. Neste estágio fica claro a intersecção de questões de mercado e a necessidade de coordenação de uma cadeia de commodities, mesmo que diferenciado. Autores como Bryant e Goodman (2004) analisam o desenvolvimento do apelo da ética para a formação de um mercado diferenciado.

\section{METODOLOGIA}

Esta pesquisa caracterizada como um estudo exploratório-descritivo, feito através de um estudo bibliométrico sobre as características e o volume de publicações sobre 
o tema "Fair Trade", baseando-se em estudos já realizados no Brasil, como o de Zandoná et al. (2009).

Estudos Bibliométricos ou Bibliometria é uma série de métodos usados para estudar ou medir textos e informação, que lidam com a medição das propriedades dos documentos, sendo usados nas ciências da informação, utilizando análises quantitativas e estatísticas para descrever padrões de publicação dado um campo ou corpo de uma literatura (HENDERSON; SHURVILLE; FERNSTROM, 2009; JALAL; BISWAS; MUKHOPADHYAY, 2009; BIBLIOMETRICS, 2009)

Este estudo bibliométrico utiliza duas abordagens. A análise dos artigos em sí e a análise das citações recebidas por eles. A primeira consiste na análise dos conteúdos inerentes ao artigo, como ano de publicação, autores, tipos de documento, entre outros. A segunda trata das citações em que os artigos são mencionados. Para ela, os autores se basearam no trabalho de Zandoná et al. (2009), utilizando o método $\boldsymbol{h}$ - $\boldsymbol{b}$, criado por Banks no seu artigo An extension of the Hirsch index: Indexing scientific topics and compounds (BANKS, 2008).

Segundo Banks (2008), esse método serve para auxiliar os pricipiantes a descobrir quanto interesse existe em tal tópico e quanto trabalho já foi alcançado nessa área. Um pesquisador novo em um tema pode ter as seguintes perguntas: Quanto já foi feito nesse tópico? Esse é um "tópico quente"? Quantas pessoas estarão interessadas na minha tese ou pesquisa? Com a riqueza de informação hoje em dia, pode ser difícil responder essas questões sem fazer uma enorme quantidade de procura na literatura.

Banks (2008) propõe que uma resposta simples e rápida pode ser obtida utilizando-se o índice $\boldsymbol{h}-\boldsymbol{b}$. O método $\boldsymbol{h}-\boldsymbol{b}$ é uma extensão do índice Hirsch, criado em 2005. Ele é feito através do volume de citações de um determinado tópico no período analisado (no caso deste trabalho, 2000 a 2009), listados em ordem ordem decrescente de citações. Ele equivale ao número de posição do artigo que tenha recebido um número de citações igual ou imediatamente maior à sua posição no ranking. Com o índice $\boldsymbol{h}-\boldsymbol{b}$ em mãos, deve-se obter o índice $\boldsymbol{m}$, que serve pra determinar o nível de interesse no tópico. Deve-se dividir o índice $\boldsymbol{h}-\boldsymbol{b}$ pelo número de anos do período analisado, ou seja, desde data da publicação mais antiga analisada.

R. eletr. estrat. neg., Florianópolis, v.4, n.2, p. 223-244, jun./dez. 2011 
Banks (2008) propõe ainda seguinte interpretação do índice $\boldsymbol{m}$. Um valor entre 0 e 0,5 representa uma combinação a qual é provavelmente de interesse dos pesquisadores em um determinado campo de estudo, cujo campo é uma comunidade menor. Um valor entre 0,5 e 2 representa um componente que provavelmente é um "tópico quente" (emergente) como área de pesquisa, onde a comunidade é muito grande. Um valor igual ou acima de 2 reflete uma mistura única, a qual alcança conseqüências para além de sua própria área de pesquisa, sendo possivelmente um tópico de aplicação prática ou com características únicas.

Esse trabalho utiliza duas bases distintas. Uma base com artigos no cenário internacional e outra no cenário nacional. As bases de dados internacionais utilizadas no presente artigo foram coletadas no catálogo eletrônico da THOMSON ISI (http://isiknowledge.com), sendo "Fair Trade" a palavra utilizada para fazer a busca na faixa de tempo de 2000 a 2009 no catálogo eletrônico.

A pesquisa resultou em 322 artigos encontrados. Depois de coletadas, as publicações foram analisadas e classificadas com base em diversas características, sendo elas: quantidade de publicações por ano, autor, campo específico de estudo, análise documental, fonte e análise espacial. Em um segundo passo foi identificado o índice $\boldsymbol{h}$ - $\boldsymbol{b}$ e $\boldsymbol{m}$ sobre o tema Fair Trade com base nos artigos coletados. Em seguida, foram agrupadas as 10 publicações mais citadas.

As bases nacionais seguiram o modelo de coleta das internacionais, sendo escolhida arbitrariamente uma grande base de dados, nesse caso, o Scielo (Scientific Eletronic Library Online), pesquisando somente artigos do Brasil. Alguns eventos nacionais foram utilizados, e partindo-se da perspectiva que este seria um tema da "gestão de cadeias", e da acessibilidade dos dados pesquisou-se nos anais dos seguintes eventos: EnANPAD, SIMPOI e ENEGEP. Os artigos publicados sobre o tema no Brasil são escassos, assim esse trabalho utilizou uma abordagem diferente para a análise nacional. Os nove artigos encontrados foram colocados em um quadro indicando o nome, o autor principal, o ano e a fonte. 


\section{RESULTADOS}

\subsection{RESULTADOS DA PESQUISA BIBLIOMÉTRICA INTERNACIONAL}

Partindo-se dos dados levantados, conforme tabela 1 , os resultados dessa pesquisa serão apresentados, discutidos e analisados a seguir.

tabela 1: Base do Estudo

\begin{tabular}{l|l}
\hline & No de trabalhos a partir das palavras-chave. \\
\hline Base Internacional & 322 \\
\hline Base Nacional & 9 \\
\hline Total & 331 \\
\hline
\end{tabular}

Fonte: elaborado pelos autores

\subsubsection{Análise temporal (por ano de publicação)}

O ano com maior número de publicações, foi 2009 , com $80(24,84 \%)$, seguido de 2008, com 66(20,50\%). Tais dados mostram o crescimento de publicações em Fair Trade nos últimos anos no cenário internacional. Logo após eles vem 2005 e 2007 quase empatados, com $43(13,35 \%)$ e 39(12,11\%) artigos respectivamente e temos o ano de 2006 com 30(9,32\%) publicações. As publicações nos anos anteriores a 2005 apresentam um volume bem menor, como se poderá notar no gráfico a seguir. Isso demonstra a emergência do tópico a partir desse ano, o que parece indicar que o meio cientifico está se sensibilizando com os problemas sociais. A figura 1 ilustra bem o crescimento de publicações na área. 
Figura 1-Número de artigos por ano.

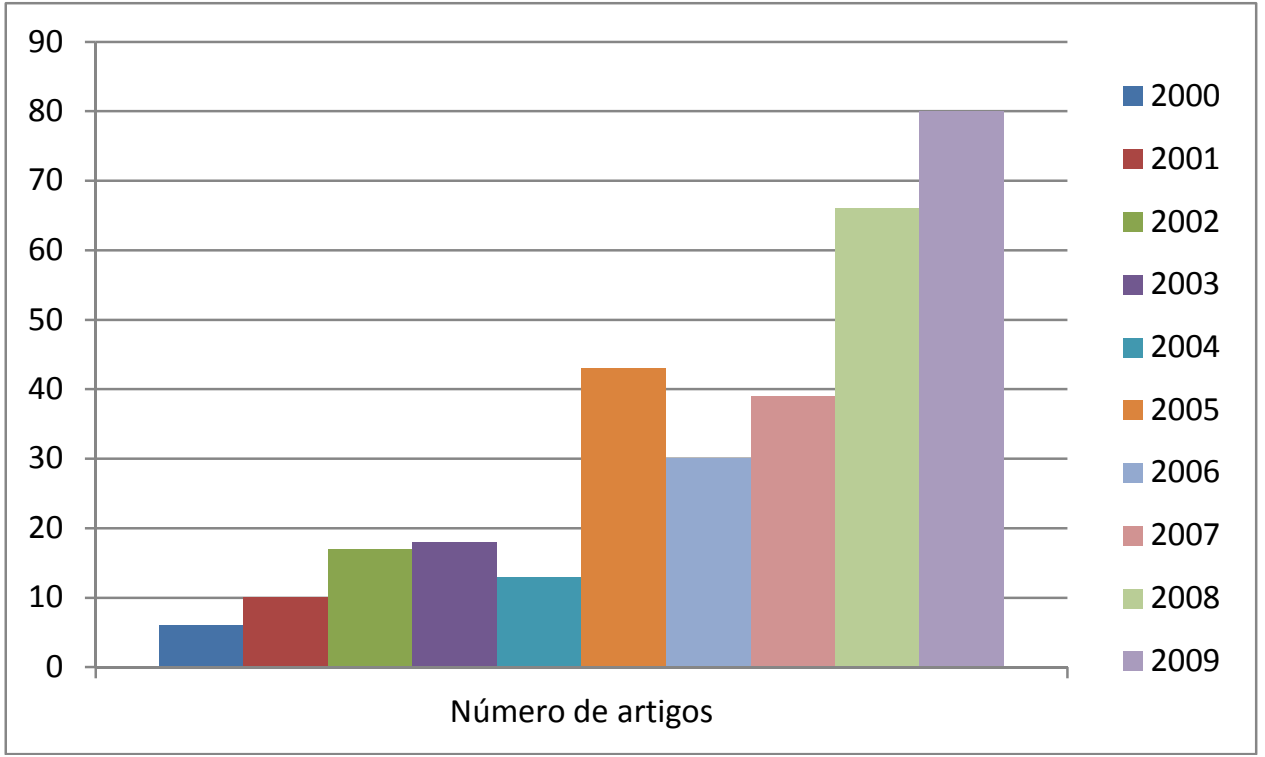

Fonte: elaborado pelos autores

\subsubsection{Análise autoral}

Nessa pesquisa, dez autores apareceram com três ou mais publicações nessa área. Foram eles: Gavin Fridell, Stefano Ponte, Ian Hudson, Alex Hughes, Tad Mutersbaugh, Laura Raynolds, Clive Barnett, Leonardo Becchetti, Martin Buttle e Nick Clarke. Os co-autores dos trabalhos deles não são considerados para esses dados. A tabela 2 faz uma relação dos autores com o maior número de publicações na área.

Tabela 2- Autores com mais publicações em FT.

\begin{tabular}{l|c}
\hline Autor & Número de publicações \\
\hline Gavin Fridell & 5 \\
\hline Stefano Ponte & 5 \\
\hline lan Hudson & 4 \\
\hline Alex Hughes & 4 \\
\hline Tad Mutersbaugh & 4 \\
\hline Laura Raynolds & 4 \\
\hline Clive Barnett & 3 \\
\hline Leonardo Becchetti & 3 \\
\hline Martin Buttle & 3 \\
\hline Nick Clarke & 3 \\
\hline
\end{tabular}

Fonte: elaborado pelos autores 


\subsection{3 Área geral de estudo e Campo específico de estudo}

Em relação à área geral de estudo, 243 publicações estão relacionadas a Ciências Sociais, 203 a Ciência e Tecnologia e 20 a Artes e Humanidades. Além disso, 5 publicações não estão inseridas em nenhuma área específica. Em relação ao campo específico de estudo, existe uma variedade bem maior, demonstrada na tabela 3 a seguir:

Tabela 3-Publicações por campo de estudo.

\begin{tabular}{l|l}
\hline Campo específico de estudo & Número de publicações \\
\hline Negócios \& Economia & 140 \\
\hline Agricultura & 68 \\
\hline Ciências Alimentícias \& Tecnologia & 61 \\
\hline Ciências Ambientais \& Ecologia & 55 \\
\hline Ciências Sociais - Outros tópicos & 47 \\
\hline Administração Pública & 41 \\
\hline Geografia & 37 \\
\hline Psicologia & 35 \\
\hline Governo \& Leis & 34 \\
\hline Sociologia & 28
\end{tabular}

Fonte: elaborado pelos autores

De todos os trabalhos, 62 não se encaixaram em nenhuma dessas categorias, portanto não estão citados nessa tabela. O campo Negócios e Economia tem o maior número de publicações, indicando que o Fair Trade é visto também como uma prática econômica e não somente como um mecanismo de ajuda social.

\subsubsection{Análise documental}

Nessa categoria, percebeu-se que, das 322 publicações analisadas, 207(64,28\%) foram escritas em formato de artigo, demonstrando a grande predominância desse tipo de documento. No restante tem-se 52 resenhas, 34 na categoria Arte e Literatura, 34 livros, 28 meetings, 15 editoriais, 13 patentes, 5 notícias, 2 cartas e 2 abstracts. A figura 2 a seguir esquematiza melhor essa distribuição. 
Figura 2-Tipos de publicações

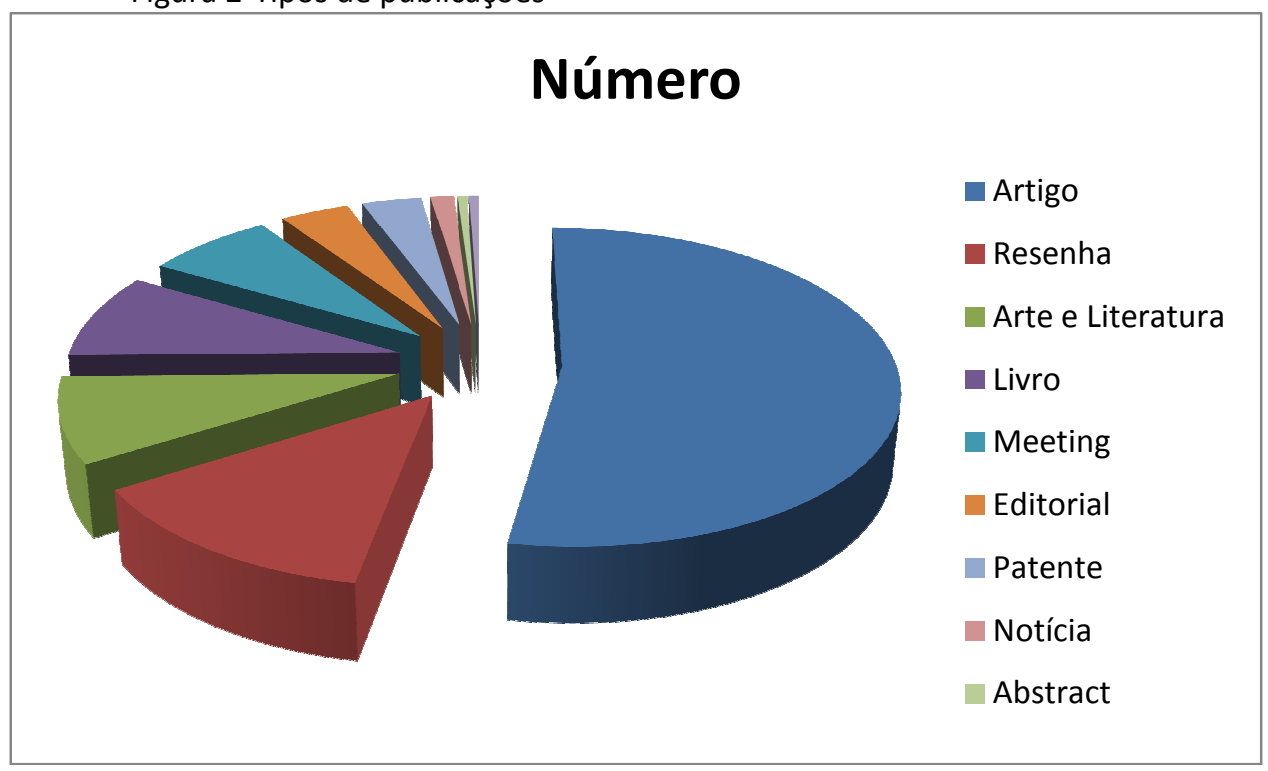

Fonte: Elaborado pelos autores

As nove (9) fontes que mais publicaram sobre Fair Trade estão listadas na tabela 4 a seguir. A principal fonte, Journal of Business Ethics, é uma publicações de negócios, ressaltando novamente o Fair Trade como uma prática econômica e não puramente social.

Tabela 4-Fontes dos artigos

\begin{tabular}{l|l}
\hline \multicolumn{1}{c|}{ Fonte } & Número de Publicações \\
\hline JOURNAL OF BUSINESS ETHICS & 31 \\
\hline SUSTAINABLE DEVELOPMENT & 10 \\
\hline JOURNAL OF RURAL STUDIES & 9 \\
\hline WORLD DEVELOPMENT & 9 \\
\hline AGRICULTURE AND HUMAN VALUES & 7 \\
\hline GEOFORUM & 6 \\
\hline PROGRESS IN HUMAN GEOGRAPHY & 6 \\
\hline CANADIAN JOURNAL OF DEVELOPMENT STUDIES - REVUE & \multicolumn{2}{|c}{} \\
CANADIENNE D ETUDES DU DEVELOPPEMENT & 4 \\
\hline ENVIRONMENT AND PLANNING A & 4 \\
\hline JOURNAL OF AGRICULTURAL \& ENVIRONMENTAL ETHICS & 4 \\
\hline
\end{tabular}

Fonte: elaborado pelo autor

\subsubsection{Estado da Arte e Consolidação Teórica}


$\mathrm{Na}$ pesquisa sobre o estado da arte busca-se identificar os 10 principais artigos sobre o tema, para isto procura-se os 10 mais citados (tabela 5)

Tabela 5-As 10 publicações mais citadas na base pesquisada

\begin{tabular}{|c|c|c|}
\hline Posição & Autor, ano, título e fonte & $\mathrm{N}^{\circ}$ de citações \\
\hline $1^{\circ}$ & $\begin{array}{l}\text { Raynolds, L. T. (2002). "Consumer/producer links in Fair Trade coffee } \\
\text { networks." Sociologia Ruralis 42(4): 404-+. }\end{array}$ & 69 \\
\hline $2^{\circ}$ & $\begin{array}{l}\text { Renard, M. C. (2003). "Fair Trade: quality, market and conventions." } \\
\text { Journal of Rural Studies 19(1): 87-96. }\end{array}$ & 60 \\
\hline $3^{\circ}$ & $\begin{array}{l}\text { Ponte, S. and P. Gibbon (2005). "Quality standards, conventions and } \\
\text { the governance of global value chains." Economy and Society 34(1): } \\
1-31 .\end{array}$ & 55 \\
\hline $4^{\circ}$ & $\begin{array}{l}\text { Goodman, M. K. (2004). "Reading fair trade: political ecological } \\
\text { imaginary and the moral economy of fair trade foods." Political } \\
\text { Geography 23(7): 891-915. }\end{array}$ & 48 \\
\hline $5^{\circ}$ & $\begin{array}{l}\text { Bryant, R. L. and M. K. Goodman (2004). "Consuming narratives: the } \\
\text { political ecology of 'alternative' consumption." Transactions of the } \\
\text { Institute of British Geographers 29(3): 344-366. }\end{array}$ & 44 \\
\hline $6^{\circ}$ & $\begin{array}{l}\text { Koletzko, B., S. Baker, et al. (2005). "Global standard for the } \\
\text { composition of infant formula: Recommendations of an ESPGHAN } \\
\text { Coordinated International Expert Group." Journal of Pediatric } \\
\text { Gastroenterology and Nutrition 41(5): 584-599. }\end{array}$ & 39 \\
\hline \multirow[t]{2}{*}{$7^{\circ}$} & $\begin{array}{l}\text { Bacon, C. (2005). "Confronting the coffee crisis: Can Fair Trade, } \\
\text { organic, and specialty coffees reduce small-scale farmer } \\
\text { vulnerability in northern Nicaragua?" World Development 33(3): } \\
497-511 .\end{array}$ & 36 \\
\hline & $\begin{array}{l}\text { Watts, D. C. H., B. Ilbery, et al. (2005). "Making reconnections in } \\
\text { agro-food geography: alternative systems of food provision." } \\
\text { Progress in Human Geography 29(1): } 22-40 .\end{array}$ & 36 \\
\hline $9^{\circ}$ & $\begin{array}{l}\text { Taylor, P. L. (2005). "In the market but not of it: Fair trade coffee and } \\
\text { forest stewardship council certification as market-based social } \\
\text { change." World Development 33(1): } 129-147 \text {. }\end{array}$ & 33 \\
\hline $10^{\circ}$ & $\begin{array}{l}\text { Rice, R. A. (2001). "Noble goals and challenging terrain: Organic and } \\
\text { fair trade coffee movements in the global marketplace." Journal of } \\
\text { Agricultural \& Environmental Ethics 14(1): 39-66. }\end{array}$ & 30 \\
\hline
\end{tabular}

Fonte: Elaborado pelo autor

\subsubsection{Quão quente é o tópico. Resultados dos Índices $h$-b e $m$}

A seguir, pretende-se identificar os índices $\boldsymbol{h}-\boldsymbol{b}$ e $\boldsymbol{m}$, como discutidos na parte metodológica. No cálculo dos índices $\boldsymbol{h}$ - $\boldsymbol{b}$ e $\boldsymbol{m}$ foram considerados os 322 artigos encontrados e o período de tempo de 10 anos (2000-2009), encontrando-se os seguintes números:

\section{1 para o índice $\boldsymbol{h}-\boldsymbol{b}$ e}


FAIR TRADE (COMÉRCIO JUSTO) COMO UM “TÓPICO QUENTE” INTERNACIONAL E SUA ABORDAGEM NO BRASIL José Carlos Lazaro Silva-Filho - Flavio Leandro Batista de Moura Cantalice

2,1 para o índice $\boldsymbol{m}$.

Então, pode-se analisar esses números da seguinte forma: as 21 primeiras publicações receberam no mínimo 21 citações cada no período analisado (hot topic?)

Sendo $\boldsymbol{m}=2,1$, isto significa que A quantidade mínima de citações por ano por referência mais citada, dentro do período analisado é 2,1.

Analisando mais especificamente o índice $\boldsymbol{m}$, de acordo com Banks (2006), um valor acima de 2 é o limite entre que seria um "tópico quente" e uma mistura única, a qual alcança conseqüências para além de sua própria área de pesquisa, sendo possivelmente um tópico de aplicação prática ou com características únicas.

O Fair Trade, com 2,1 se encaixa nessa definição apesar de passar razoavelmente perto do limiar. Deduz-se, através de índice $\mathbf{m}$ e da análise temporal que o tema Fair Trade (Comércio Justo) está em pleno crescimento nesses últimos anos.

\subsection{RESULTADOS DA PESQUISA BIBLIOMÉTRICA NACIONAL}

Como o número de artigos no cenário nacional utilizando tanto o termo "Fair Trade" como "Comércio Justo" foi bem limitado, não faria sentido dividir a classificação em várias etapas como foi feito com os artigos internacionais. O quadro 6 a seguir classifica os artigos em Nome, Autor Principal, Ano e Fonte. é importante destacar também que um dos artigos é repetido, "A global value chain aprroach to the fair trade system: a case study from small honey producers in RS", de Luciana Marques Vieira, que foi publicado no SIMPOI e na Revista de Economia e Sociologia Rural (incluído nesse artigo através do Scielo). Isso deixa um total de nove artigos na área, entre os anos de 2000 e 2009. Um número bem pífio em comparação aos 322 encontrados na data base internacional. Isso indica que o tema não é muito discutido nacionalmente, ou pelo menos não com a mesma denominação, ou seja, existem alguns artigos que tratam de atividades comerciais similares ao Fair Trade, porém utilizando denominações e métodos diferentes. Como esse artigo não se propõe a examinar tais atividades, pode ser considerada uma limitação desse artigo e uma oportunidade para um artigo posterior. 
Quadro 1-Artigos nacionais

\begin{tabular}{|l|l|l|l|}
\hline Artigo & Autor principal & Ano & Fonte \\
\hline Marketing Social e as ONGD's de Comércio Justo & $\begin{array}{l}\text { João Augusto } \\
\text { Ramos e Silva }\end{array}$ & 2001 & Enanpad \\
\hline $\begin{array}{l}\text { Conflitos na Construção de Sentido na Casa Verde: Uma } \\
\text { Experiência de Uso Conjunto das Teorias Institucional e da } \\
\text { Estruturação na Análise Organizacional }\end{array}$ & $\begin{array}{l}\text { Andrea } \\
\text { Rodrigues }\end{array}$ & 2005 & Enanpad \\
\hline $\begin{array}{l}\text { Rótulos ambientais como ferramenta de acesso a mercados } \\
\text { de países desenvolvidos: o caso do açúcar da native } \\
\text { alimentos orgânicos }\end{array}$ & $\begin{array}{l}\text { André Pereira de } \\
\text { Carvalho }\end{array}$ & 2007 & Simpoi \\
\hline $\begin{array}{l}\text { Um estudo exploratório sobre o sistema de certificação fair } \\
\text { trade e duas possibilidades de aplicação na cadeia apícola }\end{array}$ & Tatiana Maia & 2007 & Simpoi \\
\hline $\begin{array}{l}\text { Os canais de distribuição de produtos orgânicos e éticos: um } \\
\text { estudo de caso da Eco-Citrus }\end{array}$ & $\begin{array}{l}\text { Luciana Marques } \\
\text { Vieira }\end{array}$ & 2008 & Simpoi \\
\hline $\begin{array}{l}\text { A global value chain approach to the fair trade system: a case } \\
\text { study from small honey producers in RS }\end{array}$ & $\begin{array}{l}\text { Luciana Marques } \\
\text { Vieira }\end{array}$ & 2009 & $\begin{array}{l}\text { Anais do } \\
\text { SIMPOI }\end{array}$ \\
\hline $\begin{array}{l}\text { O comércio justo e solidário é alternativa para segmentos } \\
\text { populacionais empobrecidos? Estudo de caso em Território } \\
\text { Kalunga }\end{array}$ & $\begin{array}{l}\text { Breno Aragão } \\
\text { Tiburcio }\end{array}$ & 2007 & Scielo \\
\hline $\begin{array}{l}\text { A global value chain approach to the fair trade system: a case } \\
\text { study from small honey producers in RS (repetido) }\end{array}$ & $\begin{array}{l}\text { Luciana Marques } \\
\text { Vieira }\end{array}$ & 2009 & $\begin{array}{l}\text { Caroline } \\
\text { (vommen } \\
\text { Scielo) }\end{array}$ \\
\hline $\begin{array}{l}\text { Comércio e direitos humanos: rumo à coerência } \\
\text { Fonte: eng }\end{array}$ & 2005 & Scielo \\
\hline
\end{tabular}

Fonte: elaborado pelo autor

\section{DISCUSSÃO DOS RESULTADOS}

Percebemos, pelas nossas análises, que Comércio Justo (ou Fair Trade) é um tema em grande crescimento devido ao seu caráter universal. Somente o ano de 2009 apresentou 80 trabalhos na área, e a expectativa é que tenha aumentado em 2010. O objetivo principal desse trabalho é ajudar os pesquisadores que estiverem começando na área a terem um conhecimento de como ela se desenvolveu nos últimos anos no cenário internacional, como também os principais autores que publicaram na área e os trabalhos que mais foram citados, ou seja, os que têm mais relevância. 
Além disso, com o índice $\mathbf{m}$, o iniciante na área pode saber como disse Banks (2008), quanto já foi feito na área do Comércio Justo e o quão interessadas as pessoas estarão em um possível trabalho nessa área. Uma questão levantada pelo estudo é a possibilidade da diferença considerável entre publicações internacionais e nacionais é a aparente restrição dos estudos nacionais a seguirem abordagem de uma cadeia de commodities, que seria uma evolução de uma convenção social para uma convenção de mercado. Devido a importação desta convenção de mercado sem uma consolidada convenção social antes, acaba por não ser suficiente para estruturar as cadeias.

Dessa forma, os autores esperam ter feito uma contribuição relevante para a comunidade científica e esperam incentivar os trabalhos desse tipo, seguindo o caminho de Zandoná et al (2009) .

\title{
FAIR TRADE: AS AN INTERNATIONAL HOT TOPIC AND ITS APPROACH IN BRAZIL
}

\begin{abstract}
This paper aims to analyse the concept of Fair Trade in international and Braszilian literature. Throgh the identification of the grow of thema and analyzing the possible "barriers" to the development of the theme in the Brazilian context. The authors develop two bibliometric research, a eletronic amd international one based on database of "Web of Science from ISI" and another in the brazilian publications (proceedings and journals). Although the indentification of the thma as a real emerging theme in international literature("hot topic"), the authors noted a significant gap in the Brazilian publications. The author propouses to use use the theoretical approach, that emerges fom the French social science, the "quality and conventions" aproach that are being use to understand the coordination of networks of agribusiness So, in Brazil, the Fair Trade still inscipiente because it does not comes from a "civic (moral) convention", that could be a necessary phase to achive de "market
\end{abstract}


FAIR TRADE (COMÉRCIO JUSTO) COMO UM ‘TÓPICO QUENTE” INTERNACIONAL E SUA ABORDAGEM NO BRASIL

convention", in other side de developed countries began has a civic convention and are achiving the market as a new convention, assimilated by the retails networks.

Keywords: fair Trade. Convetion Theory. Bibliometry.

\section{REFERÊNCIAS}

ADAMS, M.; RAISBOROUGH J. What Can Sociology Say About FairTrade?: Class, Reflexivity and Ethical Consumption. Sociology, Reino Unido, v. 42, n. 6, p. 1165-1182, 2008.

BANKS, M. G. An Extension of the Hirsch Index: Indexing scientific topics and compounds. Scientometrics, Holanda, v. 69, p. 161-168, 2006

BARNETT, C. et al. Consuming Ethics: Articulating the Subjects and Spaces of Ethical Consumption, Antipode, v. 37, n. 1, p. 23-45, 2005.

BECCHETTI, L. ; ADRIANI F. Fair Trade: A 'Third Generation Welfare' Mechanism to make Globalization Sustainable, Centre of International Studies on Economic Growth, Rome, 2002.

BECCHETTI, L.; HUYBRECHTS; B. The dynamics of fair trade as a mixed-form market. Journal of Business Ethics, Holanda, v. 81, n. 4, p. 733-750, 2008.

BIBLIOMETRIC, School of Information - University of Texas Disponivel em:

<http://www.ischool.utexas.edu/ palmquis/courses/biblio.html>. Acesso em 26 abr. 2010.

BRYANT, R. L. ; GOODMAN, M. K. Consuming narratives: the political acology of alternative consumption, Transactions of the Institute of British Geographers, London, v. 23, n. 3, p. 344-366, 2004.

EFTA - EUROPEAN FAIR TRADE ASSOCIATION disponível em <http://www.eftafairtrade.org/>. Acesso em: 26 fev. 2010.

FLO -FAIR TRADE LABELLING ORGANIZATIONS INTERNATIONAL, Generic Fairtrade Trade Standards, 2009. Disponível em:

<http://www.fairtrade.net/fileadmin/user_upload/content/GTS_Aug09_EN.pdf>. Acesso em: 26 abr. 2010. 
FAIR TRADE (COMÉRCIO JUSTO) COMO UM ‘TÓPICO QUENTE” INTERNACIONAL E SUA ABORDAGEM NO BRASIL José Carlos Lazaro Silva-Filho - Flavio Leandro Batista de Moura Cantalice

FLO - FAIR TRADE LABELLING ORGANIZATIONS INTERNATIONAL, Generic Fairtrade Standards: Flo Prohibited Materials List, 2007. Disponível em:

$<$ http://www.fairtrade.net/fileadmin/user_upload/content/FLO_Prohibited_Materials_List_ Dec_2007_EN.pdf>. Acesso em: 26 abr. 2010.

FLO - FAIR TRADE LABELLING ORGANIZATIONS INTERNATIONAL:

<http://www.fairtrade.net/> Acesso em 26 fev. 2010.

HENDERSON, M; SHURVILLE, S. ; FERNSTROM, K. The quantitative crunch: The impact of bibliometric research quality assessment exercises on academic development at small conferences. Campus-Wide Information Systems, v. 26, n. 3, p.149-167, $2009 .$.

HIRSCH, J. E. An index to quantify an individual's scientific research output. PNAS, EUA, v. 102, n. 46, p. 165-169, 2005.

INSTITUTO BIODINÂMICO, Sistema De Certificação Ecosocial IBD: Programa IBD Para Relações Justas Em Comércio De Produtos Orgânicos Com Certificação Socioambiental, 2009. Disponível em:

<http://www.ibd.com.br/downloads/dirleg/Diretrizes/8_1_3_IBD_Diretriz_EcoSocial_8aEdic ao_2009.pdf $>$. Acesso em 26 abr. 2010.

INSTITUTO BIODINÂMICO: <http://www.ibd.com.br/> Acesso em 26 abr. 2010.

JALAL, S. K. ; BISWAS S. C. ; MUKHOPADHYAY P. Bibliometrics to webometrics Information Studies, India, v. 15, n. 1, p. 3-20, 2009.

JONES, P. ; COMFORT, D ; HILLIER, D. A Case Study of Local Food and its routes to market in the UK. British Food Journal, Reino Unido, v. 106, n. 4, p. 328-335, 2004.

KRIER, J. M. 2005, Fair Trade in Europe 2005 : Facts and Figures on Fair Trade in 25 European Countries. Bélgica: Fair Trade Advocacy Office em Bruxelas, 2005.

MAIA, T.; VIEIRA, L. M.; WILK, E. O. Um estudo exploratório sobre o sistema de certificação Fair Trade e suas possibilidades de aplicação na cadeia apícola. Em: SIMPÓSIO DE ADMINISTRAÇÃO DA PRODUÇÃO, LOGÍSTICA E OPERAÇÕES INTERNACIONAIS, 2007, Rio de Janeiro. Anais Eletrônicos... Rio de Janeiro, 2007..

MASELAND, R.; VAAL A. D. How fair is fair trade?, De Economist, Holanda, v. 150, n. 3, p. 251-272, 2002. MOORE ,G. The Fair Trade Movement: Parameters, Issues and Future Research, Journal of Business Ethics, v. 53, n. 1, p. 73-86, 2004.

NICHOLLS, A.; OPAL, C. FairTrade: Market-Driven Ethical Consumption. London: SAGE Publications, 2005. 
RAYNOLDS, L. T. Consumer/Producer Links in Fair Trade Coffee Networks, Sociologia Ruralis, Europa, v. 42, n. 4, p. 404-424, 2002.

RENARD, M. C. Fair Trade: quality, market and conventions, Journal of Rural Studies, v. 19, n. 1, p. 87-96, 2003.

ZANDONÁ, L.; GOMES, C. M.; MADRUGA, L. R. R. G. Green Marketing: uma Análise da Emergência Temática. Em ENCONTRO NACIONAL DE GESTÂO E MEIO AMBIENTE, IX, 2009, Anais... Fortaleza: UNIFOR, 2009. 